\title{
Effect of Alternation of Aging and Seawater Erosion on Properties of Rubber Material Used in Lead Rubber Bearing
}

\author{
Yanmin $\mathrm{Li}^{1,2}$, Guifeng $\mathrm{Zhao}^{2,3}$, Yuhong $\mathrm{Ma}^{1,2, *}$ and Rong Liu ${ }^{1,2}$ \\ ${ }^{1}$ Earthquake Engineering Research \& Test Center, Guangzhou University, Guangzhou, 510405, China \\ ${ }^{2}$ Guangdong Key Laboratory of Earthquake Engineering \& Applied Technique, Guangzhou, 510405, China \\ ${ }^{3}$ School of Civil Engineering, Guangzhou University, Guangzhou, 510006, China \\ ${ }^{*}$ Corresponding Author: Yuhong Ma. Email: myhzth@gzhu.edu.cn
}

Received: 12 July 2021 Accepted: 15 October 2021

\begin{abstract}
An artificially accelerated alternation of aging and seawater erosion test of rubber materials used in lead rubber bearing (LRB) was performed, mainly to study the time-varying laws of rubber materials mechanical properties. Time-varying laws of the Mooney-Rivlin and Neo-Hookean constitutive parameters of rubber materials under the alternation of aging and seawater erosion were also analyzed. Results indicate that the rubber material mechanical properties were significantly affected by alternation of aging and seawater erosion. Hardness and elongation stress increased exponentially with test time. And 120 days after the test, the hardness increased by $14 \%$, the maximum percentage increase in stress of $124.76 \%$ occurred at $100 \%$ constant elongation and the minimum percentage increase in stress of $68.32 \%$ occurred at $300 \%$ constant elongation; Tensile strength and elongation at break decreased by $44.96 \%$ and $53.09 \%$. Besides, constitutive parameters of Mooney-Rivlin and Neo-Hookean all changed greatly with test duration. Finally, time-varying laws of constitutive parameters were verified by comparing the simulated and experimental results of the lead rubber bearing's stiffness. Research results are of great significance to the seismic performance research and life-cycle performance analysis of offshore traffic engineering such as cross-sea bridges and bridges in the marine environment.
\end{abstract}

\section{KEYWORDS}

Alternation of aging and seawater erosion; lead rubber bearing; rubber material properties; constitutive parameters; time-varying law

\section{Introduction}

With the rapid development of China's economy, seismic isolation and reduction technologies have been used in the construction of sea-spanning bridges and other offshore transportation projects. These technologies ensure the seismic safety of the resulting structures. As a key component of seismic mitigation and isolation technologies, rubber bearings are an important part of bridge superstructures and substructures, which is the weak part in bridge seismic design though. In addition, considering the longterm complex and harsh marine corrosion environment in which offshore engineering structures, such as sea-crossing bridges, are built, the performance of the rubber isolation bearing used in these structures would degrade easily, which will affect the seismic performance of the structures in which they are used. 
Rubber is one of the important materials used in lead rubber isolators. Therefore, it is important to study the degradation law and the time-varying law of the constitutive parameters of rubber material used in lead rubber isolators.

The literature includes many reports on the durability of rubber materials. Abmalek et al. [1] studied the mechanical properties of a natural rubber (NR) tire soaked in seawater for 42 years and found that the elongation at the break of the inner and outer tires decreased. Mott et al. [2] carried out air aging and seawater aging tests on NR materials. And failure strain was used to monitor the extent of degradation. $\mathrm{Gu}$ et al. [3] studied the aging performance of NR materials and high-damping rubber material used in bridge bearings under the action of several different deterioration factors. And their results indicated that thermal oxygen aging caused the rubber material to harden, which was the dominant factor leading aging. Itoh et al. [4-7] conducted a series of experimental studies on NR blocks and high-damping rubber materials. Based on the test results and experimental phenomena, they developed a model to predict the aging characteristics of natural rubber bearings (LNR) and found that thermal oxygen aging was the main factor influencing the aging high-damping rubber material. Ha-Anh et al. [8] conducted the aging test on chloroprene rubber material, summarized the change rule of the Mooney-Rivlin model constant with aging time, noting that the model constant influences the aging of rubber material. Ma et al. $[9,10]$ studied the performance degradation law of NR materials and high-damping rubber materials under aging, sea erosion and seawater dry-wet cycles. Their results showed that these factors considerably influence the hardening of rubber materials and the deterioration of its mechanical properties. Liu et al. [11] conducted an accelerated thermal aging test on nitrile rubber (NBR) to explore its aging performance in terms of morphology, crosslinking density, mechanical properties and chemical morphology and so on. Their findings showed that the surface damage to NBR was uneven, and the aging condition was the most severe in the void edge area. Zhao et al. [12] studied the continuous stress relaxation of NBR in air and nitrogen medium at temperatures of $125^{\circ} \mathrm{C}$ and $165^{\circ} \mathrm{C}$ and discussed the effect of gas medium, temperature and initial strain on stress relaxation in the material. Chaabane et al. [13] present a constitutive model for the prediction of the thermally driven network degradation by considering a collection of chemical and physical changes occurring inside the rubber-filler material system.

Ulu et al. [14] investigated the fatigue properties of filled natural rubber in seawater environment by uniaxial fatigue and crack propagation experiments, and analysed the damage by scanning electron microscopy.

Rubber isolation bearings are widely used in buildings, bridges and other structures. Finite-element analysis serves as a method for simulation and analysis of the performance of rubber bearings, offshore bridges and other maritime traffic engineering structures. The accuracy of finite-element simulation is closely related to the accuracy of the constitutive model parameters of the rubber material in question. Many researchers have studied the application of the constitutive model parameters of rubber material and methods to determine their values. Huang et al. [15] used the ANSYS software application to analyse and compare the Mooney-Rivlin model and the Yeoh model for rubber materials. Zuo et al. [16,17] theoretically derived a formula to determine the Mooney-Rivlin constitutive coefficient of rubber material with the same hardness with a small deformation range. Li et al. [18] studied the constitutive parameters of the Mooney-Rivlin model of rubber materials for rubber isolators by considering the aging time and obtained a formula to determine the deterioration of the constitutive parameters with aging time. Ma et al. [19] calculated and analysed the time-varying law of the Mooney-Rivlin constitutive parameters of NR material based on the degradation test results of NR material under aging, sea erosion and seawater drywet cycles. Yang et al. [20] used the parameters of the Neo-Hookean, Yeoh, Mooney-Rivlin and Ogden constitutive models fitted by conducting uniaxial tensile tests to investigate the tensile mechanical properties of hydroxyl-terminated polybutadiene (HTPB) coating. Based on the basic theory of finite deformation, Sang et al. [21] analysed the hyperelastic mechanical properties of rubber material under 
basic loads and discussed the influence of the Mooney-Rivlin and Neo-Hookean model parameters on the behaviour of the material $\mathrm{B}$.

In sum, the current research on the performance degradation of rubber materials mainly focuses on air aging, thermal oxygen aging, seawater corrosion and other discrete factors, as well as performance changes after a period of aging. Early days, our research group carried out experimental research on the mechanical properties of rubber material used in LNR and high-damping rubber bearing (HDR) under complex marine environment conditions such as seawater dry-wet cycles. In this paper, the time-varying law of the material properties and the constitutive parameters of the rubber material used in lead rubber bearing (LRB) under the alternating action of aging and seawater erosion are studied.

\section{Introduction to Rubber Material Test}

Given the position of rubber bearings in the cross-sea bridges, they are vulnerable to marine environmental factors, such as sea water splash, typhoons, huge waves and alternate sunny and rainy weather. In addition, considering the limitations of the existing laboratory equipment, the practicability of testing and other factors, we used a test method that involves artificial high-temperature accelerated seawater immersion and drying aging to study lead rubber bearings and their rubber material processed in the same batch. Parameters of artificial seawater were shown in Table 1. Besides, one hundred and twenty-three rubber sheets measuring $150 \mathrm{~mm} \times 116 \mathrm{~mm} \times 2 \mathrm{~mm}$ (length $\times$ width $\times$ thickness) were prepared and used to study the time-varying law of the properties and constitutive parameters of rubber material under the alternation of aging and seawater erosion condition. And the processing, manufacturing and performance testing of rubber materials used in LRB were entrusted to Chengdu Xinzhu Road \& Bridge Machinery Co., Ltd. (China). And the main components of rubber materials used in LRB are natural rubber SCR WF (polyisoprene) $80 \mathrm{phr}$, styrene butadiene 1502 (polybutadiene styrene) $20 \mathrm{phr}$, sulphur powder (sulfur) $1.5 \mathrm{phr}$, stearic acid $1 \mathrm{phr}$, zinc oxide $5 \mathrm{phr}$, antioxidant $4 \mathrm{phr}$, vulcanization accelerator (N-cyclohexyl-2-benzothiazole sulfonamide and 2, 2'-dibenzothiazole disulfide) $2 \mathrm{phr}$, carbon black (carbon) $45 \mathrm{phr}$, petroleum resin (C9) $8 \mathrm{phr}$. Besides, the physical properties of rubber materials used in LRB should meet the requirements of Table 10 in reference [22]. Artificially accelerated alternation of aging and seawater erosion test were applied to the rubber material and lead rubber isolation bearings simultaneously in the same environment. The influence of alternation of aging and seawater erosion on the properties and constitutive parameters of the rubber material were studied. Photographs of the placement of the test samples are shown in Figs. 1 and 2. Test parameters were determined by referring to $[9,10]$, and test temperature was set $80^{\circ} \mathrm{C}$, actual service environment temperature was set $20^{\circ} \mathrm{C}$, the time ratio of aging and seawater erosion was set $2: 1$, the speedup ratio of test was 376, which can be calculated by formula (1) in reference [19]. Therefore, subjecting the specimens to one day of alternation of aging and seawater erosion test can be equivalent to 1 year of the specimens' service life in the actual environment.

Table 1: Parameters of artificial seawater solution [25]

\begin{tabular}{llllll}
\hline Composition of artificial seawater & $\mathrm{NaCl}$ & $\mathrm{MgCl}_{2}$ & $\mathrm{Na}_{2} \mathrm{SO}_{4}$ & $\mathrm{CaCl}_{2}$ & Total amount \\
\hline Content $(\mathrm{g} / \mathrm{kg})$ & 23.5 & 5 & 4 & 1.1 & 33.6 \\
Chloride ion content $(\mathrm{g} / \mathrm{kg})$ & 14.261 & 3.737 & - & 0.704 & 18.7 \\
\hline
\end{tabular}




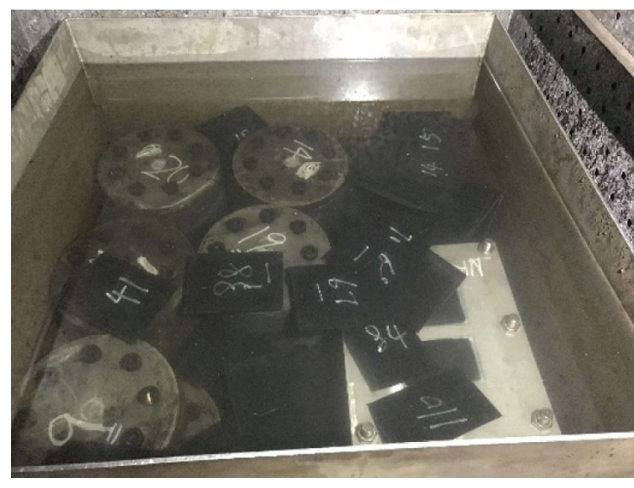

Figure 1: Samples soaking in seawater

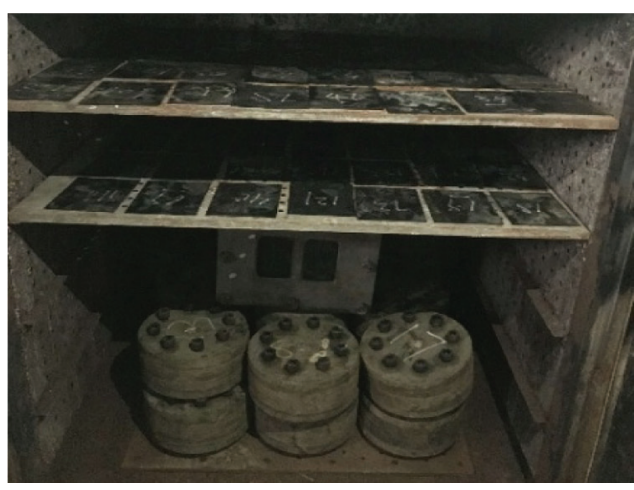

Figure 2: Samples drying

Detail test method was as following: (1) Specimens were first soaked for one day in artificial seawater in the aging box at $80^{\circ} \mathrm{C}$; (2) Then, the specimens were dried for two days in aging box at $80^{\circ} \mathrm{C}$ without artificial seawater; (3) Test was conducted the above cycle till to test end. In addition, three specimens were sampled every three days, amounting to a total of 41 instances of sampling during 120-day test period. And each rubber specimen was cut into three dumbbells (see Fig. 3) to test the rubber materials' hardness, tensile stress at a given tensile elongation ratio of $30 \%, 40 \%, 50 \%, 100 \%, 150 \%, 200 \%, 250 \%$ and $300 \%$, Elongation at the break and Tensile strength. Changes in the appearance of the rubber sheets during the test are shown in Fig. 4. And the performance test equipment of rubber material is shown in Fig. 5. Hardness of rubber material was tested in accordance with ISO 7619-1: 2004 [23], namely that after the dumbbell sample is cut off from each rubber sheet, the three samples are stacked up to a total thickness of $6 \mathrm{~mm}$, and the hardness is measured with a shore hardness tester (when the foot of the hardness tester is in close contact with the surface of the sample, the reading shall be carried out after the indicator is stable for 3 s). Its tensile mechanical properties were tested according to ISO 37: 2005 [24], the dumbbell sample is symmetrically clamped on the upper and lower clamps of the tensile testing machine, so that the tensile force is evenly distributed on the cross section, and the testing machine is started until the dumbbell sample is broken (during test, the moving speed of the gripper is $(500 \pm 50) \mathrm{mm} / \mathrm{min}$, and the change curves of the sample length and force are continuously monitored and recorded.).

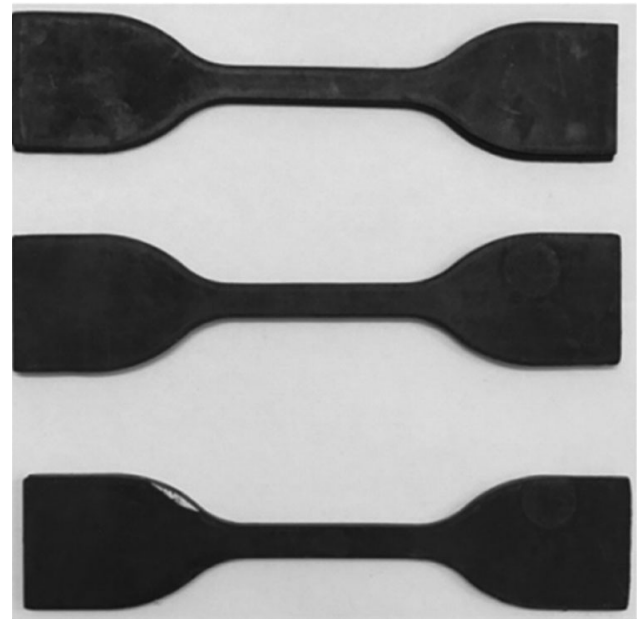

Figure 3: Dumbbell specimens

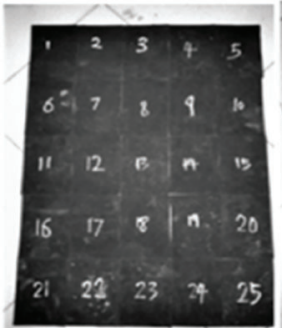

$0-21$ day

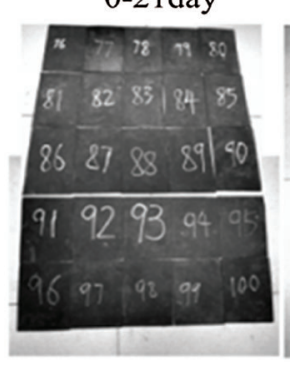

73-96day

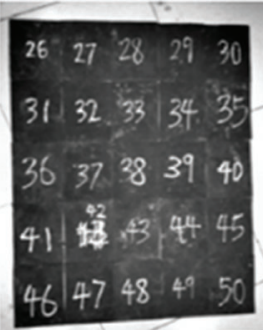

22-45day

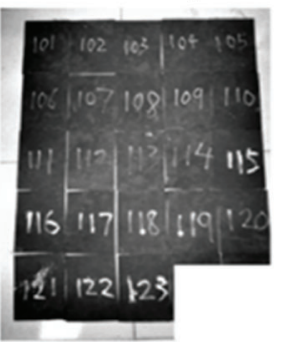

97-120day

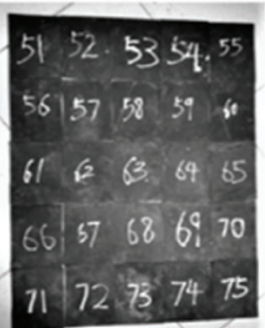

46-72day

Figure 4: Appearance of specimens during test 


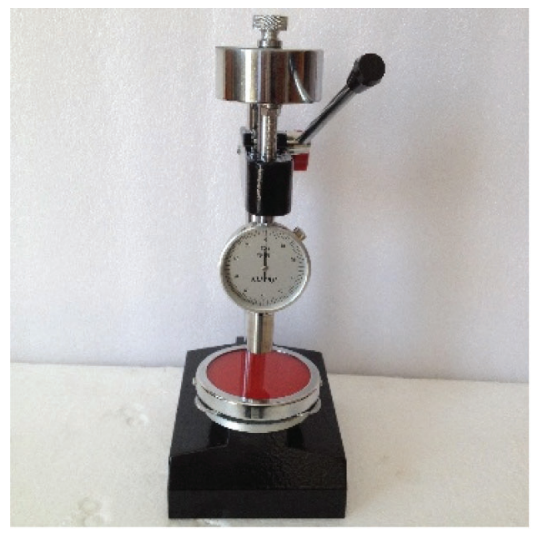

(a)

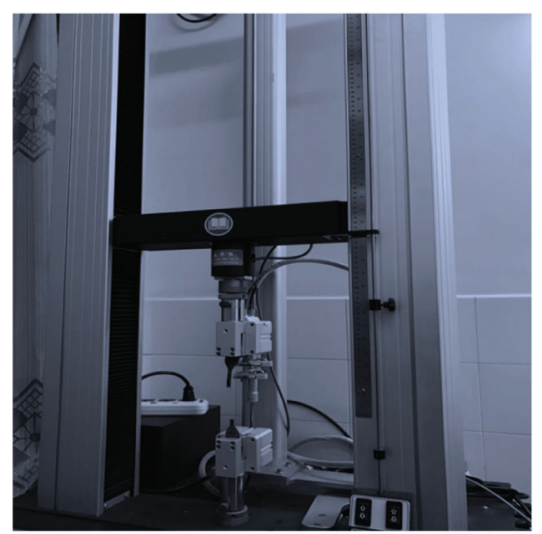

(b)

Figure 5: Performance test equipment of rubber material (a) hardness tester (b) tensile testing machine

Fig. 4 shows that the lustre of the rubber sheets gradually decreased, and touch sampling indicated that the rubber sheets gradually hardened. This result indicates that aging of rubber sheets sample is caused by seawater drying and wetting cycles. Increasing amounts of salt and rust can also be observed on the surface of the rubber sheets, which may be ascribed to corrosion of the steel plate of the lead rubber bearings placed in the same test environment.

\section{Analysis of Rubber Material Performance Test Results}

Three pieces of rubber sheets were sampled every 3 days in this study, and three dumbbell samples were cut from each rubber sheet to test their mechanical properties. The average value of the test results of nine dumbbell samples taken from each rubber sheet was considered as the final test result for analysis. In this study, the test cycle lasted 120 days, and 123 pieces of the rubber sheets, namely 369 dumbbell samples, were cut for the relevant performance tests. The results of the mechanical properties test are as follows.

\subsection{Hardness}

Hardness is an important index that affects the stiffness of rubber material. In this study, we used the shore hardness scale. To eliminate the influence of initial errors, the ratio of the hardness value $H_{\mathrm{r}}(t)$ corresponding to seawater drying and wetting cycles time $t$ the initial hardness value $H_{\mathrm{r}}(0)$ before the test was defined as the hardness ratio. The value range of $t$ in the full text and all formulae are the same: $0 \leq t \leq 120$. The hardness ratios $\left(H_{\mathrm{r}}(t) / H_{\mathrm{r}}(0)\right)$ of the rubber material obtained after the 120 -day test period (equivalent to 120 years of service in the actual environment) are shown in Fig. 6. The timevarying law of the hardness ratio of the rubber material subjected to the alternating action of aging and seawater erosion was then obtained by fitting the relevant test data (see formula in Fig. 6).

Fig. 6 shows that the hardness ratio of the rubber material increases exponentially as the test time increases, which may be caused by the cross-linking density of rubber increased due to the alternating action of aging and seawater erosion. After test durations of 60 and 120 days, the hardness ratio increased by $9.1 \%$ and $14 \%$, respectively. Therefore, the alternating action of aging and seawater erosion have a considerable effect on the hardness of the rubber material used in the lead rubber bearings tested herein. Moreover, the increase in hardness in the first 60 days was faster than that in the next 60 days. There was also a certain deviation between the fitting and experimental values of the hardness ratio of the rubber material, and the maximum and average deviations were $5.04 \%$ and $1.4 \%$, respectively. Thus, it can be concluded that the fitting accuracy was good, and formula in Fig. 6 can be used to reasonably reflect the 
time-varying law of the hardness ratio of the rubber material in lead rubber bearings subjected to the alternation of aging and seawater erosion.

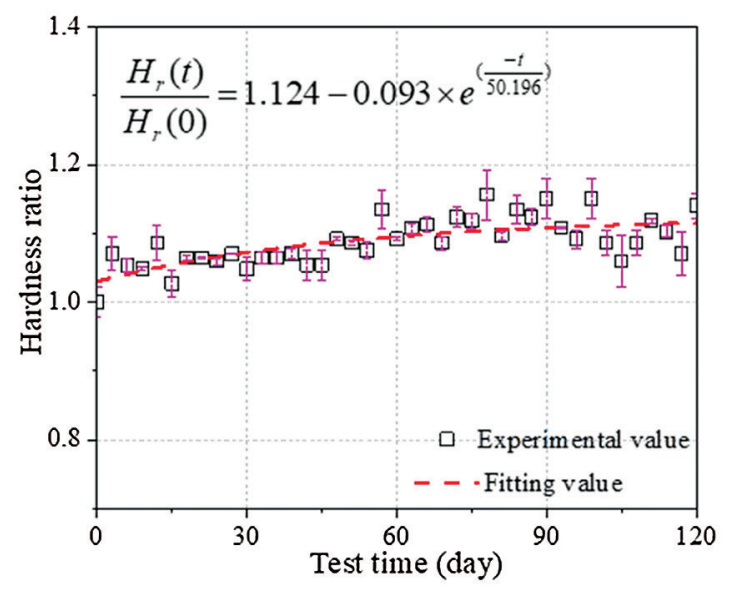

Figure 6: Effects of alternating action of aging and seawater erosion on hardness ratio of rubber material

\subsection{Stress under Constant Elongation}

In addition to hardness, stress under constant elongation is an important index of the stiffness of rubber materials. In this research, 30\%, 40\%, 50\%, 100\%, 150\%, 200\%, 250\% and 300\% tensile strain were applied in the test of stress under constant elogation of rubber material, and the test method was given in reference [24]. Similar to the above definition of the hardness ratio in Part 2.1, the ratio of stress at constant elongation $\mathrm{Se}_{\gamma}(t)$ corresponding to the test time t of the seawater drying and wetting cycles to the initial stress at constant elongation $\mathrm{Se}_{\gamma}(0)$ before the test was defined as the stress ratio at constant elongation. The test results of stress ratio at constant elongation are shown in Fig. 7. In addition, the time-varying law of stress ratio at constant elongation under alternating action of aging and seawater erosion was fitted according to the relevant test data (see formula in Fig. 7). The deviation between the fitted and experimental values and the range of increase of each stress at constant elongation after the test durations of 60 and 120 days are given in Table 2.

Fig. 7 shows that the stress ratio at constant elongation of rubber material increases exponentially with the alternating of aging and seawater erosion test duration. The reason why may be that the cross-linking density of rubber increases, which increases the external force required for certain deformation of rubber, so as to increase the constant tensile stress. Besides, the growth rate was rapid in the early stages and stable in the final stage, which was similar to the change rule of the hardness ratio with test duration. But the increasing trend of stress ratio at constant elongation was more significant. According to Table 2, the stress ratio at constant elongation of the rubber material used herein increased greatly in the first 60 days than in the latter 60 days, and all the growth amplitudes after test for 60 days of stress ration with various elongation were more than $60 \%$. In addition, the magnitude of the increase in the stress ratio at $100 \%$ elongation was the highest and that of the stress ratio at $300 \%$ elongation was the least, which may be ascribed to the fact that the increase of crosslinking density of rubber was the highest at $100 \%$ elongation strain. The fitting value of the stress ratio at constant elongation agreed well with the experimental value. The maximum deviation was mostly within $15 \%$, and only the maximum deviation of the stress ratio at $100 \%$ elongation was more than $20 \%$. Formula in Fig. 7 can approximately describe the time-varying law of the relationship between stress ratio at constant elongation of the rubber material and duration of the alternation of aging and seawater erosion. Considering the convenience of practical application, by combining the relevant test data and formula in Fig. 7, the relationship between the modulus of stress $\mathrm{Se}$ of the rubber material, alternating action of aging and seawater erosion duration $t$ and tensile strain $\gamma$ was obtained, as can be seen in formula (1). 
$\operatorname{Se}(t, \gamma)=\frac{0.719+0.0171 t-0.0001 t^{2}+0.3435 \gamma}{1+0.0004 t-0.8047 \gamma+0.3123 \gamma^{2}-0.0433 \gamma^{3}}$

The average deviation between the fitting value calculated using formula (1) and the experimental value of stress at constant elongation was $7.77 \%$. Formula (1) can basically describe the change law of stress at constant elongation of the rubber material with tensile strain and alternating action of aging and seawater erosion time, which can provide a theoretical basis and serve as a reference in the development of practical engineering applications.

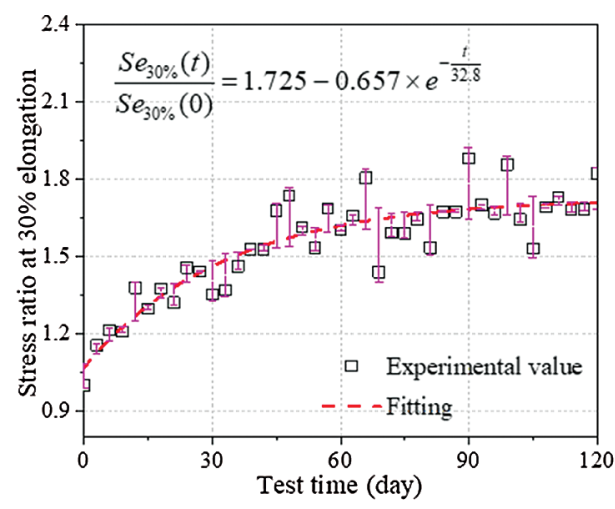

(a)

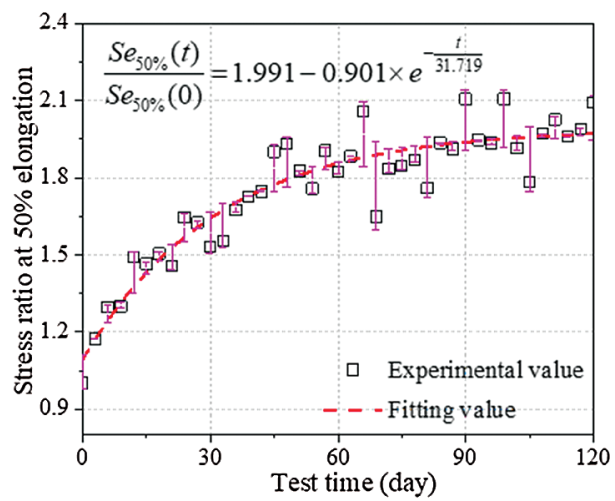

(c)

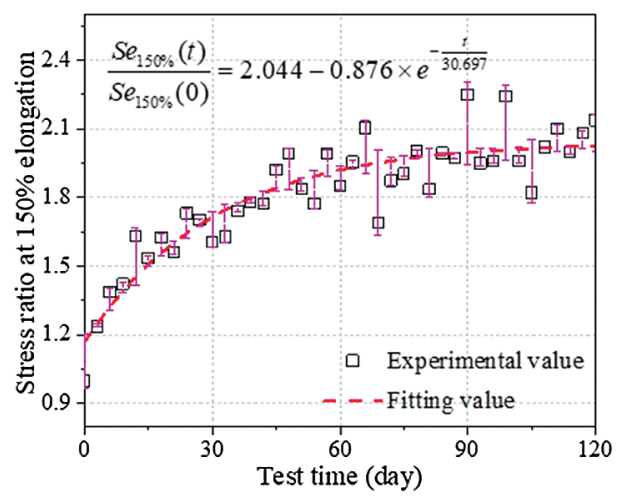

(e)

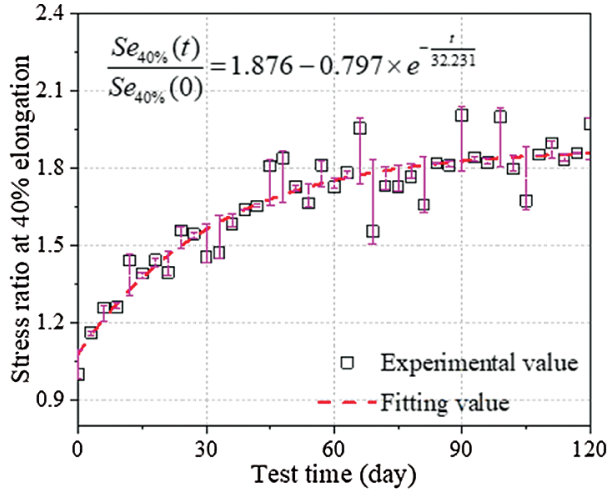

(b)

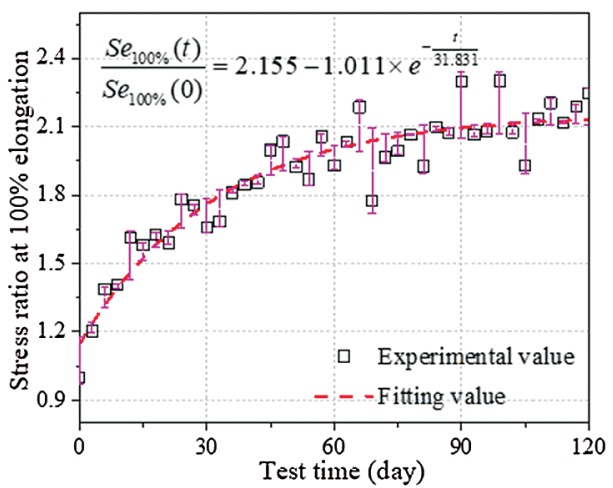

(d)

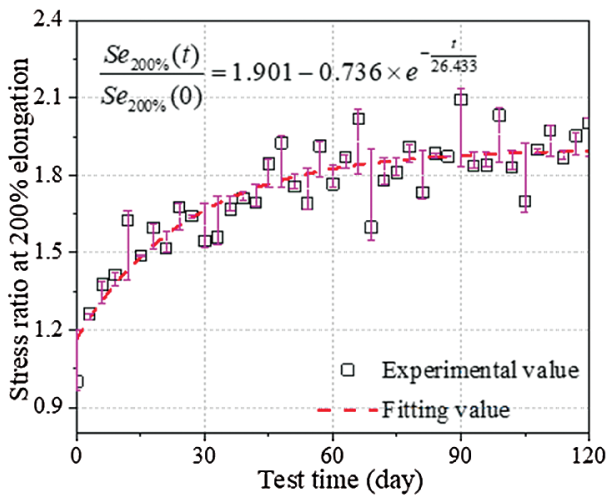

(f)

Figure 7: Continued 


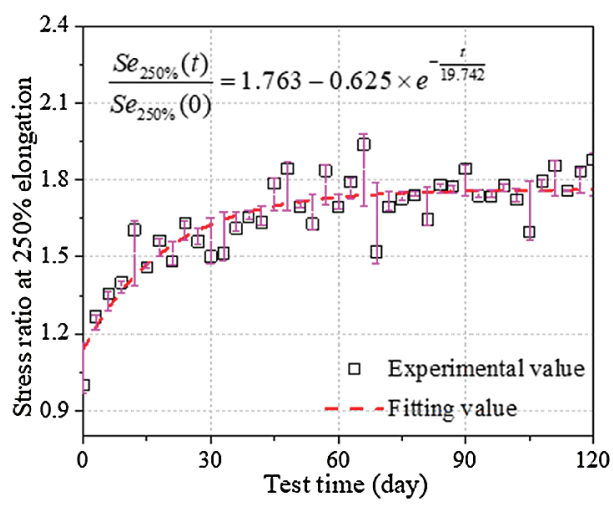

(g)

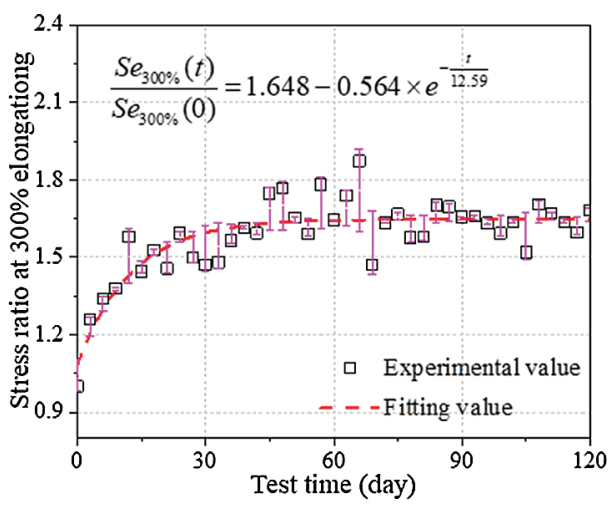

(h)

Figure 7: Effect of alternating action of aging and seawater erosion on the stress ratio at constant elongation of rubber material (a) stress ratio at 30\% elongation (b) stress ratio at 40\% elongation (c) stress ratio at 50\% elongation (d) stress ratio at $100 \%$ elongation (e) stress ratio at $150 \%$ elongation (f) stress ratio at $200 \%$ elongation $(\mathrm{g})$ stress ratio at $250 \%$ elongation $(\mathrm{h})$ stress ratio at $300 \%$ elongation

Table 2: Deviations of stress ratio at constant elongation for rubber material and its growth amplitude

\begin{tabular}{lllll}
\hline \multirow{2}{*}{ Test project } & \multicolumn{2}{c}{$\begin{array}{l}\text { Deviations between fitting } \\
\text { and experimental value }\end{array}$} & $\begin{array}{l}\text { Growth amplitude after } \\
\text { test for 60 days (\%) }\end{array}$ & $\begin{array}{l}\text { Growth amplitude after } \\
\text { test for 120 days (\%) }\end{array}$ \\
\cline { 2 - 4 } & & Average deviation (\%) & \\
\hline Stress ratio at & $30 \%$ & 4.2 & 60.11 & 82.03 \\
constant & $40 \%$ & 3.95 & 72.61 & 96.97 \\
elongation & $50 \%$ & 3.91 & 82.12 & 109.15 \\
& $100 \%$ & 7.71 & 93.19 & 124.76 \\
& $150 \%$ & 4.73 & 85 & 113.74 \\
& $200 \%$ & 4.8 & 76.5 & 100.74 \\
& $250 \%$ & 4.43 & 69.25 & 87.9 \\
& $300 \%$ & 3.97 & 64.42 & 68.32 \\
\hline
\end{tabular}

\subsection{Tensile Strength}

The tensile strength of rubber material is its ultimate ability to resist tensile failure, and it is one of the important indexes of rubber material. The test value of the tensile strength of the rubber material sample used herein is shown in Fig. 8. The time-varying law of tensile strength ratio with test duration was obtained by fitting the test values of tensile strength of rubber specimens, and supposing that the fitting formular is written as: $\frac{T S(t)}{T S(0)}=a+b \times \mathrm{e}^{-\frac{t}{c}}$, where $\mathrm{a}, \mathrm{b}$, and $\mathrm{c}$ are the parameters during the fitting produce. Then imposing the constraint $\mathrm{a} \geq 0$, and the optimization algorithm: Levenberg Marquardt calculation and analysis shows that a can ensure that the formular $\frac{T S(t)}{T S(0)}>0$ (i.e., with reasonable behavior) within the interval [0 0.1]. When $\mathrm{a}=$ 0.01 is listed in this paper, the formula is shown in Fig. 8. Then, a comparison between the fitted values and test values is shown in Fig. 8. 
JRM, 2022, vol.10, no.6

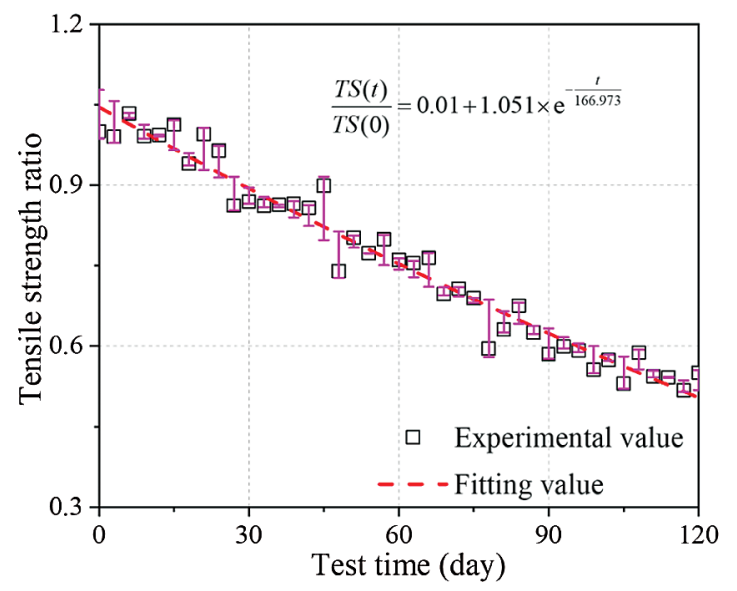

Figure 8: Effect of the alternating action of aging and seawater erosion on the tensile strength ratio of rubber material

Fig. 8 shows that the tensile strength ratio of rubber material decreased exponentially as the duration for which alternating action of aging and seawater erosion are applied increased, and the magnitudes of decrease were $23.97 \%$ and $44.96 \%$, respectively, for test durations of 60 and 120 days. The reason why may be that the alternating of aging and seawater erosion lead to the increase of internal molecular weight and the decrease of cross-linking bond energy. These results indicate that the alternating action of aging and seawater erosion significantly affected the tensile strength of the rubber material. Moreover, there was a certain deviation between the values fitted using Formula in Fig. 8 and the experimental values. The average deviation was $3.47 \%$. The rubber material studied in this paper was used in lead rubber bearing. The rubber bearing's testing deviation of horizontal and vertical stiffness are $15 \%$ and $30 \%$, respectively, which stated in specification $[22,26,27]$. Thus, the deviations within this range were evaluated as within the acceptable range.

\subsection{Elongation at Break}

Elongation at break is an important index of rubber materials. A comparison of the experimental and the fitted values is shown in Fig. 9. The time-varying law of elongation at break ratio with seawater dry-wet cycle duration is given by Formula in Fig. 9.

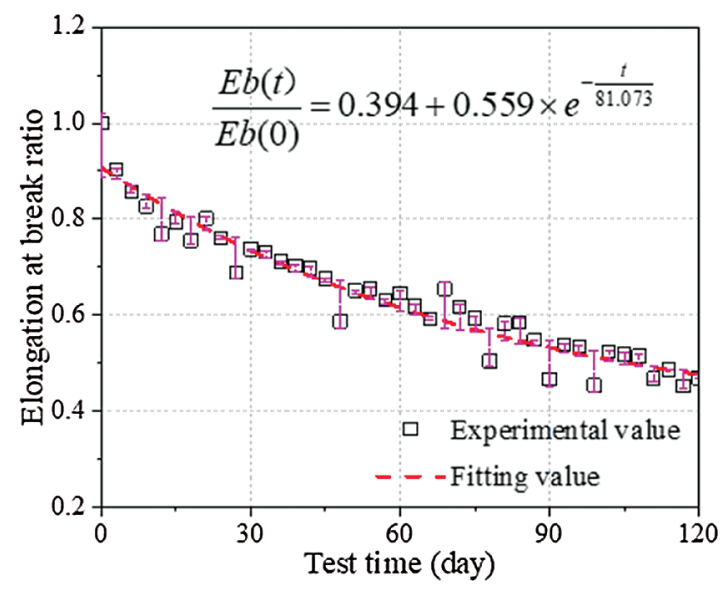

Figure 9: Effects of alternation of aging and seawater erosion on the elongation at break ratio of rubber material 
Fig. 9 indicates that the elongation at break ratio of the rubber material decreased exponentially as the seawater dry-wet cycle duration increased, which may be caused by that the interaction force and network structure between rubber molecules and fillers has been destroyed, the ability of rubber to resist external forces has been weakened and then the elongation at break has been decreased, under alternating aging and seawater erosion. This is similar to the change trend of tensile strength. Moreover, after the test durations of 60 and 120 days, the elongation at break of the rubber material decreased by $35.45 \%$ and $53.09 \%$, respectively. These findings indicate that the seawater dry-wet cycle duration significantly influenced the elongation at break. Moreover, the average deviations between the values fitted using Formula in Fig. 9 and the experimental values was $4.23 \%$, which indicates that the fitted values agreed well with the test values. Therefore, Formula in Fig. 9 was considered the time-varying law of elongation at break under alternating action of aging and seawater erosion in this work.

In sum, the effect of alternating action of aging and seawater erosion on the mechanical properties of the rubber material used in lead rubber bearings was found to be significant. The alternating cycles caused deterioration of the material's mechanical properties, possibly by effecting changes in the internal structure of the material, such as its molecular weight and crosslinking density. The findings of this study are similar to the findings of our previous studies $[9,10]$.

\section{Time-Varying Law of Constitutive Parameters of Rubber Material}

The mechanical properties of rubber materials change greatly under the alternation of aging and seawater erosion, so the corresponding material constitutive model parameters may also change. In addition, the accuracy and rationality of the constitutive parameters of rubber materials are closely related to the accuracy of the finite-element simulation analysis results of rubber bearings and bridge structures. Based on the above research results pertaining to the degradation law of the mechanical properties of the rubber material used in lead rubber bearings under the alternation of aging and seawater erosion, the time-varying law of the constitutive parameters of rubber material was studied to provide a theoretical basis for further and comprehensive research on the performance degradation of rubber isolation bearings in marine environment and to provide a reference for prediction of the lifecycle performance of offshore isolation structures. The tensile stress-strain curve of the rubber material measured during the test is illustrated in Fig. 10.

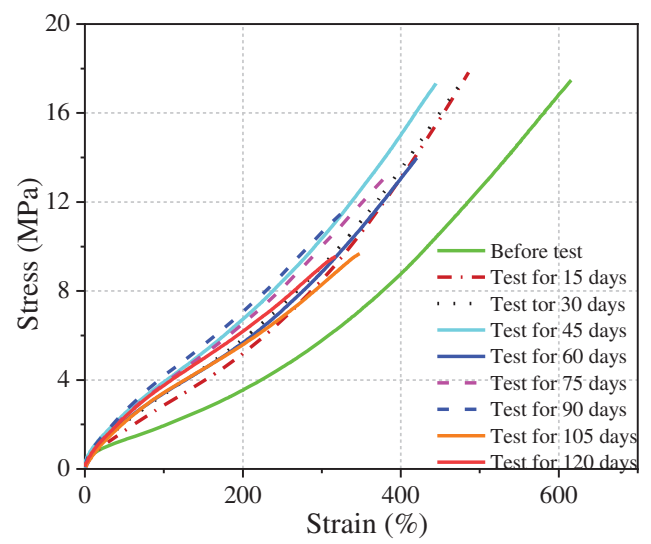

Figure 10: Tensile stress-strain curve of the rubber material during the alternation of aging and seawater erosion test 
Fig. 10 shows that for the same test duration, the growth speed of tensile stress of rubber material first increased and then decreased as the test time increased, peaking at 90 days. However, for the same tensile strain, the tensile stress of the rubber material decreased as alternation of aging and seawater erosion duration increased, and the tensile failure stress decreased as the test duration increased. The tensile failure stress decreased from 17.47 to $13.98 \mathrm{MPa}$ and $9.55 \mathrm{MPa}$ after testing for 60 and 120 days, respectively, which amounted to decreases of $19.98 \%$ and $45.33 \%$. Under the same tensile stress, the tensile strain decreased as the test duration increased, and the rate of decrease peaked at 90 days. Moreover, the ultimate strain decreased from $615.6 \%$ to $420.3 \%$ and $317.8 \%$ after 60 and 120 days of testing, respectively, and the corresponding decrease percentages were $31.72 \%$ and $48.38 \%$. Consequently, the effect of alternating action of aging and seawater erosion on the tensile stress-strain curve of the rubber material was noticeable.

Although many constitutive models for rubber materials are available, the widely used Mooney-Rivlin and Neo-Hookean constitutive models were selected and their constitutive parameters calculated. These parameters were then substituted into the finite-element model of the lead rubber bearing to analyse the basic mechanical properties of the bearing. Finally, the simulation and experimental performance results of the lead rubber bearing were compared, and the feasibility and rationality of the constitutive parameters were verified.

\subsection{Determination of Mooney-Rivlin Constitutive Parameters}

The Mooney-Rivlin constitutive parameters $C_{10}$ and $C_{01}$ of the rubber material were calculated using the least-squares fitting method [18,19] based on the stress-strain and stress at constant elongation. The variation of $C_{10}$ and $C_{01}$ with the test duration is shown in Fig. 11. The time-varying laws of the Mooney-Rivlin constitutive parameters were obtained by fitting their calculated values (see formulae in Fig. 11). A comparison between the fitted and calculated values is shown in Fig. 11.

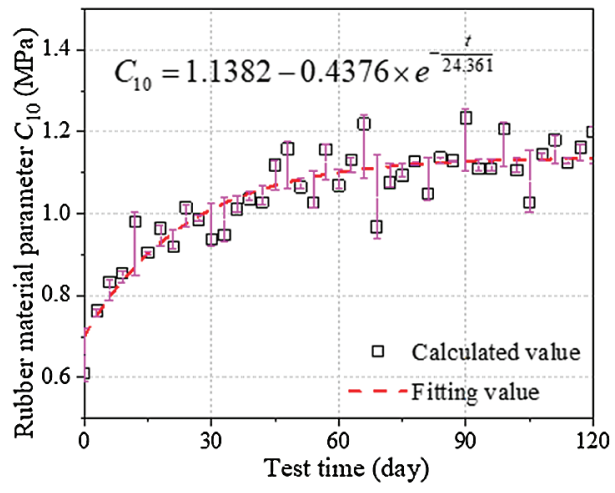

(a)

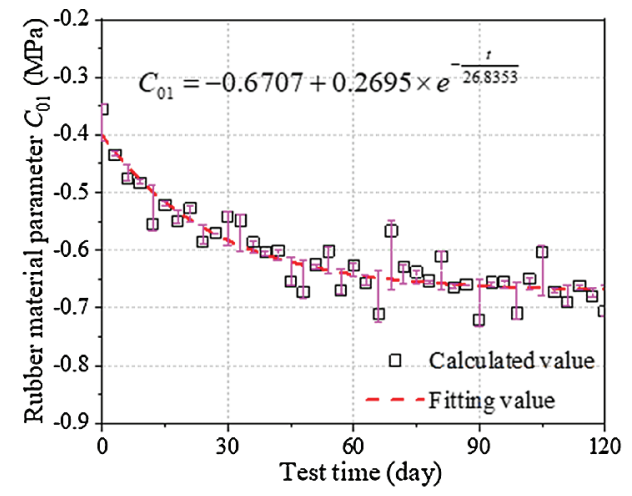

(b)

Figure 11: Influence of alternating action of aging and seawater erosion on the rubber material constant (a) $C_{10}$ (b) $C_{01}$

According to Fig. 11, the rubber material parameter $C_{10}$ increased exponentially as the alternating action of aging and seawater erosion duration increased, whilst the parameter $C_{01}$ decreased exponentially as the test time increased. Moreover, deviations were observed between the calculated and fitted values of the rubber material parameters, but these deviations were acceptable. The maximum deviations of the material parameters $C_{10}$ and $C_{01}$ were less than $15 \%$, and the average deviations were less than $5 \%$. Hence, Formulae in Fig. 11 were used as the time-varying laws of the Mooney-Rivlin constitutive parameters with alternating action of aging and seawater erosion duration in this study. Moreover, the 
Mooney-Rivlin constitutive function expression considering the alternation of aging and seawater erosion duration can be obtained by substituting the time-varying formula in Fig. 11 into Mooney-Rivlin constitutive model's function expression, as in formula (2).

$$
\begin{aligned}
W= & \left(1.1382-0.4376 \times e^{-\frac{t}{24.361}}\right) \times\left(I_{1}-3\right)+\left(-0.6707+0.2695 \times e^{-\frac{t}{26.8353}}\right) \times\left(I_{2}-3\right) \\
& +\frac{1}{D_{1}} \times(J-1)^{2}
\end{aligned}
$$

where $t$ is alternation of aging and seawater erosion test duration (days), $I_{1}$ and $I_{2}$ are deformation tensor invariants, $D_{1}$ is the compressibility of the rubber material and $J$ is the elastic volume ratio, the same below in formula (6).

\subsection{Determination of Neo-Hookean Constitutive Parameters}

The formula for variation of hardness with the duration of seawater drying and wetting cycles was obtained by fitting the experimental hardness values, see formula (3):

$H_{r}=69.66-5.78 \times e^{-\frac{t}{50.2}}$

The deviations between the values calculated using formula (3) and the experimental values were small, and the maximum deviation was less than 5\%. Hence, formula (3) can be considered accurate. Therefore, the shear modulus value of the rubber material corresponding to different hardness values was calculated by means of interpolation based on the shear modulus and hardness values given in reference [22], and the hardness value was calculated using formula (3). The trend of change in the shear modulus $G$ of the rubber material with the duration of alternation of aging and seawater erosion was then obtained by fitting the shear modulus value with various cycle durations (see Fig. 12 and formula (4)). The NeoHookean constitutive parameter $C_{N H 1}$ (the parameter $C_{10}$ of the Neo-Hookean constitutive model is expressed as $C_{N H 1}$ in this paper to distinguish it from the Mooney-Rivlin constitutive parameter $C_{10}$ ) for various durations was obtained based on the shear modulus values calculated using formula (4), see Fig. 13. The fitted time-varying law based on the calculated values in Fig. 13 is expressed as formula (5).

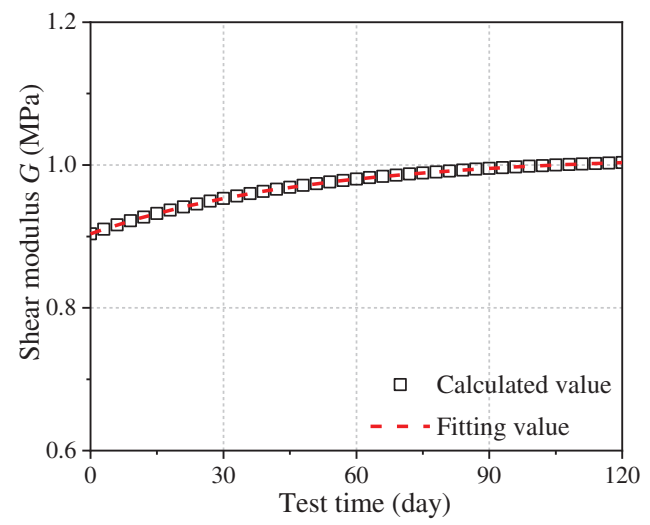

Figure 12: Effect of alternating action of aging and seawater erosion on the shear modulus $G$ of the rubber material 


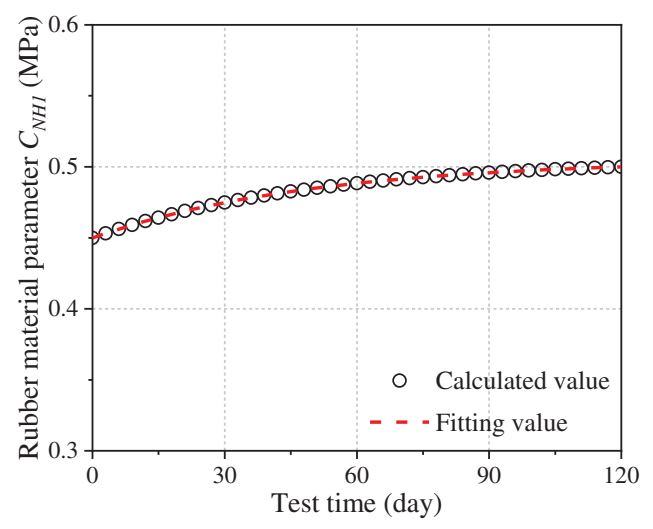

Figure 13: Effect of alternating action of aging and seawater erosion on the rubber material constant $C_{N H 1}$

Figs. 12 and 13 indicate that both the shear modulus G and the Neo-Hookean constitutive parameter $C_{N H 1}$ of the rubber material increased exponentially as the duration of the alternation of aging and seawater erosion increased, and their fitted values agreed well with their calculated values. This indicates the rationality of formulas (4) and (5).

$G=1.01-0.11 \times e^{-0.02 \times t}$

$C_{N H 1}=0.505-0.055 \times e^{-0.02 \times t}$

Similarly, the obtained time-varying formula (5) of the Neo-Hookean constitutive parameter was substituted into its function expression, and the Neo-Hookean constitutive function expression considering the duration of the seawater drying and wetting cycles is expressed as formula (6).

$W=\left(0.505-0.055 \times e^{-0.02 t}\right) \times\left(I_{1}-3\right)+\frac{1}{D_{1}} \times(J-1)^{2}$

\section{Validation of Constitutive Parameters}

To validate the effectiveness of the proposed constitutive parameter's change law under alternation of aging and seawater erosion, a finite element model of LRB is developed using Abaqus. LRB's diameter, shear modulus and overall height is $220 \mathrm{~mm}, 0.8 \mathrm{MPa}$ and $103.4 \mathrm{~mm}$. Besides, the total heights of inner steel plates, inner rubber and cover steel plates are $21,42.4$ and $40 \mathrm{~mm}$, the lead core's diameter is $20 \mathrm{~mm}$. In addition, in the finite element model the steel plate is assumed to be elastoplastic with Young's modulus $2 \times 10^{11} \mathrm{~Pa}$, and a Poisson's ratio of 0.3 . The rubber parameters under various test time are determined by Sections 4.1 and 4.2. And we assume that the bonding strength between the steel and rubber can be ignored, and that the rubber layer and the internal steel plate share one node in this model. And the rotational degree of freedom of LRB is not considered in this finite element, the bottom of LRB is constrained by $\mathrm{X}, \mathrm{Y}$, and $\mathrm{Z}$ degrees of freedom, which can reasonably reflect the real situation of LRB's performance test. Additionally, the element types of steel plate and the rubber in this finite element model are $\mathrm{C} 3 \mathrm{D} 8 \mathrm{R}$ and $\mathrm{C} 3 \mathrm{D} 8 \mathrm{H}$, respectively. And the finite element grid is divided according to 'the principle of coin' [21]. The Mooney-Rivlin and Neo-Hookean constitutive model parameters corresponding to the various durations of alternation of aging and seawater erosion were calculated and substituted into the finite-element model of the lead rubber bearing (see Fig. 14) to analyse the horizonal and vertical stiffness of the bearing. Thereafter, the simulated and experimental values of the horizontal and vertical stiffness of the lead rubber bearing at various test time points were compared to verify the accuracy and rationality of the time-varying law of these two constitutive model parameters. Considering 
that the performance of the lead rubber bearing was tested only at $0,15,30,45,60,75,90,105,120$ days during the test period and the vertical stiffness data at 105 and 120 days were not collected correctly, only the existing experimental results and the corresponding simulation results were compared and presented in this paper.

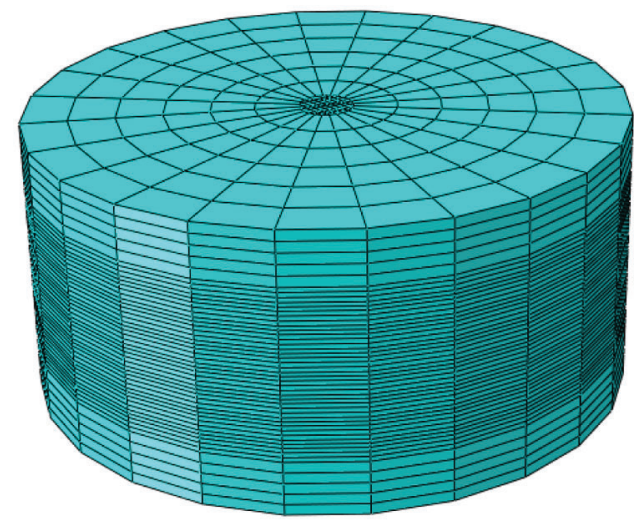

Figure 14: Finite element model of lead rubber bearing

\subsection{Validation of Mooney-Rivlin Constitutive Parameters}

It was pointed out in specifications $[22,26,27]$ that the reference temperature for horizontal shear performance test of rubber isolation bearing is $23^{\circ} \mathrm{C}$. And if the test temperature is not $23^{\circ} \mathrm{C}$, the experimental values shall be corrected. The test temperature in this research is not $23^{\circ} \mathrm{C}$, so the temperature correction was made for the experimental value of horizontal stiffness in this paper. And the correction formula is as follows:

$K_{h-\text { temp }}=K_{\text {h-test }} \times e^{-0.00271 \times(23-T)}$

In which, $K_{h \text {-temp }}$ is the temperature correction value of horizontal stiffness; $K_{h \text {-test }}$ is the experimental value of horizontal stiffness; $T$ is the test temperature (unit is ${ }^{\circ} \mathrm{C}$ ).

By using the above time-varying Formulae in Fig. 11, the Mooney-Rivlin constitutive parameters were calculated for different time nodes and substituted into the finite-element model to analyse the performance of the lead rubber isolation bearing. A comparison between the finite-element simulation values and the experimental values of the horizontal and vertical stiffness is presented in Fig. 15.

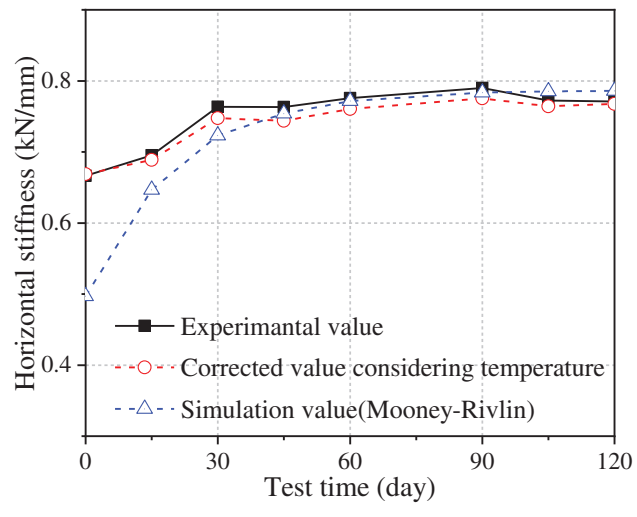

(a)

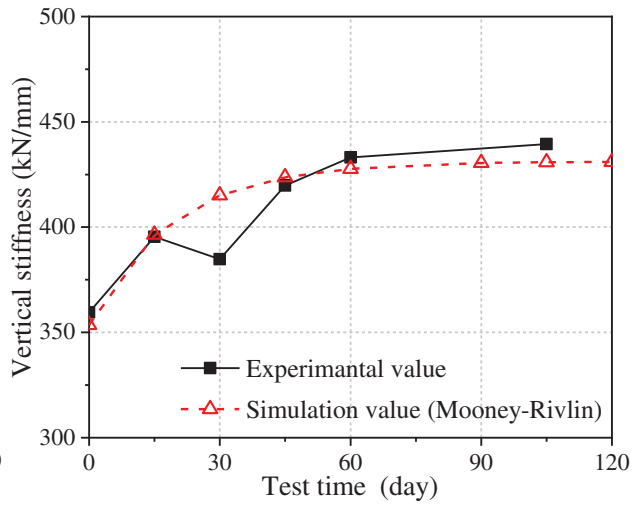

(b)

Figure 15: Comparison between the experimental value and the simulated value of stiffness in terms of the Mooney-Rivlin constitutive parameters (a) horizontal stiffness (b) vertical stiffness 
Fig. 15 shows a certain deviation between the simulated and experimental results of the horizontal and vertical stiffness of the lead rubber bearing, but the overall agreement is good. The maximum, and average deviations between the simulated values and experimental values of horizontal stiffness were $25.41 \%$, and $5.48 \%$, respectively, and those between the simulated value and the corrected value for horizontal stiffness considering the temperature were $25.7 \%$, and $5.51 \%$. The maximum, and average deviations between the simulated and experimental values of vertical stiffness were $7.86 \%$, and $2.32 \%$, respectively. This comparison indicated that the proposed time-varying formula in Fig. 11 reflected the trend of changes in the Mooney-Rivlin constitutive parameters under the alternation of aging and seawater erosion.

\subsection{Validation of Neo-Hookean Constitutive Parameter}

According to the obtained time-varying law given by formula (4), the Neo-Hookean constitutive parameter corresponding to various durations of the alternation of aging and seawater erosion were calculated and substituted into the finite-element model to analyse the performance of the lead rubber bearing. A comparison between the simulated and experimental results is shown in Fig. 16.

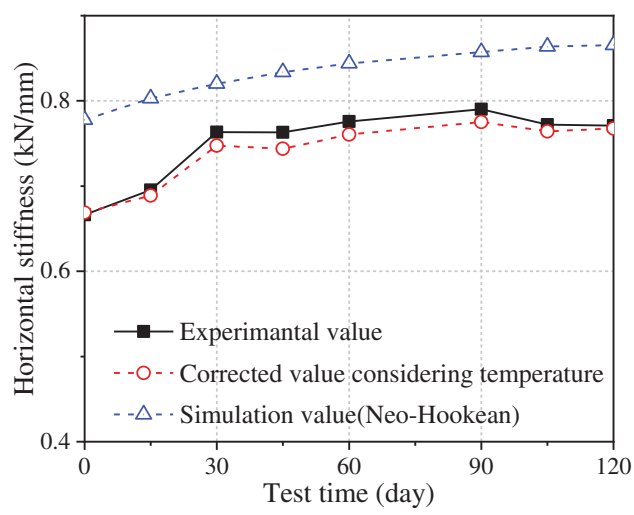

(a)

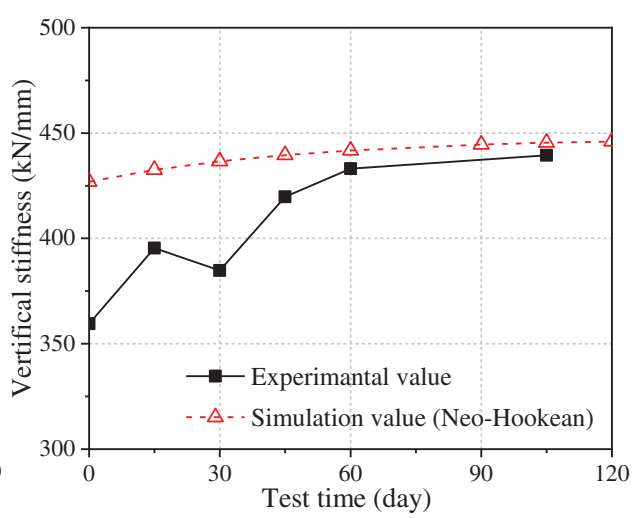

(b)

Figure 16: Comparison between the experimental value and the simulated value of stiffness by using the Neo-Hookean constitutive parameters (a) horizontal stiffness (b) vertical stiffness

Fig. 16 indicate that the simulated values, based on the above Neo-Hookean constitutive parameter, of the horizontal and vertical stiffness were consistent with the corresponding experimental values, and the comparison of vertical stiffness was better than that of horizontal stiffness. Moreover, the comparison result based on the Mooney-Rivlin constitutive parameters is better than that based on the Neo-Hookean constitutive parameters. The maximum, and average deviations between the simulated values (based on the Neo-Hookean constitutive parameter) and experimental values of horizontal stiffness were $16.7 \%$, and $11.3 \%$, respectively, and those between the simulated values and the temperature-corrected values of horizontal stiffness were $16.6 \%$, and $12.8 \%$. The maximum, and average deviations between the simulated and experimental values of vertical stiffness were $18.73 \%$, and $8.27 \%$, respectively. The proposed time-varying laws of the constitutive parameters of the rubber material can serve as a reference for finite-element simulation analysis of rubber bearings in marine environments.

\section{Conclusions}

A 120-day alternation of aging and seawater erosion test was carried out on the rubber material used in lead rubber isolation bearings. During the test, the mechanical properties of the rubber material were tested regularly, and the time-varying laws of the properties were obtained based on the test results. The timevarying formulas to determine the Mooney-Rivlin and Neo-Hookean constitutive model parameters under 
alternating action of aging and seawater erosion were proposed based on the experimental data of constant elongation stress and stress-strain and combined with the least-squares numerical analysis method. Finally, the obtained constitutive parameters were substituted into a finite-element model of the lead rubber bearing to analyse its basic mechanical properties, and the simulation results were compared with the corresponding experimental results to verify the rationality and feasibility of the proposed time-varying formulas of the constitutive parameters of the rubber material. The main conclusions are as follows:

(1) The hardness ratio of the rubber material used in the lead rubber bearing increased exponentially with the test duration. After testing for 120 days, the hardness increased by $14 \%$, and the growth rate in the first 60 days was faster than that in the subsequent 60 days.

(2) The stress ratio at constant elongation increased exponentially with the test duration, which was similar to the trend of change in the hardness ratio. Whilst the growth rate of the stress ratio at constant elongation was faster in the early stage of the test and tended to be stable in the later stage. The rate of increase of the stress ratio at 300\% elongation was only $3.9 \%$ after the test duration of 60 days. In addition, the growth rate of the stress ratio at $100 \%$ elongation was the highest, and that at the stress ratio of $300 \%$ elongation was the lowest. The results also indicate that at the same test time, the stress ratio at fixed elongation increased as the tensile strain increased, while at the same tensile strain, the stress ratio increased with the test time.

(3) Both the tensile strength and the elongation at break decreased exponentially as the duration for which the alternating action of aging and seawater erosion were applied increased. After 120 days of testing, the percentages of decrease in these parameters were $44.96 \%$ and $53.09 \%$, respectively, which indicated that the seawater drying and wetting cycles significantly affected the tensile strength and the elongation at break of the rubber material used in the lead rubber bearing.

(4) The Mooney-Rivlin constitutive parameters $C_{10}$ and $C_{01}$ increased and decreased exponentially with the duration of the alternation of aging and seawater erosion test, respectively. Moreover, the Neo-Hookean constitutive parameter $C_{N H 1}$ increased exponentially as the test duration increased. The time-varying expressions of each of the constitutive parameters are given in formula in Fig. 11 and formula (5), which can facilitate the finite-element study of similar bearing performance and the corresponding structural analyses and provide theoretical support for lifecycle performance evaluation, design and seismic performance research of rubber bearings and offshore bridges.

(5) The proposed formula for constitutive parameters are suitable for determining the Mooney-Rivlin and Neo-Hookean constitutive parameters of rubber materials used in bridge isolation bearings, which are with 120 years of service under the alternating of aging and seawater erosion.

Acknowledgement: The authors gratefully acknowledge the financial support of this work by the Guangzhou University. They also acknowledge the Earthquake Engineering Research \& Test Center.

Funding Statement: This work was supported by the Programme for National Natural Science Foundation of China (52078150, 51878196), National Key R\&D Program of China (2019YFE0112500), 2019 Guangzhou University Full-Time Graduate "Basic Innovation” Project (2019GDJC-D11).

Conflicts of Interest: The authors declare that they have no conflicts of interest to report regarding the present study.

\section{References}

1. Abmalek, K., Stevenson, A. (1986). The effect of 42-year immersion in sea-water on natural rubber. Journal of Materials Science, 21(1), 147-154. DOI 10.1007/BF01144713. 
2. Mott, P., Roland, C. (2001). Aging of natural rubber in air and seawater. Rubber Chemistry and Technology, 74(1), 79-88. DOI 10.5254/1.3547641.

3. Gu, H., Itoh, Y. (2010). Ageing behaviour of natural rubber and high damping rubber material used in bridge rubber bearings. Advances in Structural Engineering, 13(6), 1105-1113. DOI 10.1260/1369-4332.13.6.1105.

4. Itoh, Y., Gu, H. (2009). Prediction of aging characteristics in natural rubber bearings used in bridges. Journal of Bridge Engineering, 14(2), 122-128. DOI 10.1061/(ASCE)1084-0702(2009)14:2(122).

5. Itoh, Y., Gu, H., Satoh, K., Kutsuna, Y. (2006). Experimental investigation on ageing behaviors of rubbers used for bridge bearings. Doboku Gakkai Ronbunshuu A, 62(1), 176-190. DOI 10.2208/jsceja.62.176.

6. Itoh, Y., Yazawa, A., Kitagawa, T., Kitagawa, T. (2002). Study on environmental durability of rubber bearing for bridges. IABSE Symposium Melbourne, 86, 137-143. DOI 10.2749/melbourne.2002.

7. Itoh, Y., Gu, H., Satoh, K., Yamamoto, Y. (2006). Long-term deterioration of high damping rubber bridge bearing. Doboku Gakkai Ronbunshuu A, 62(3), 595-607. DOI 10.2208/jsceja.62.595.

8. Ha-Anh, T., Vu-Khanh, T. (2005). Prediction of mechanical properties of polychloroprene during thermo-oxidative aging. Polymer Testing, 24(6), 775-780. DOI 10.1016/j.polymertesting.2005.03.016.

9. Li, Y. M., Ma, Y. H., Zhao, G. F., Zhou, F. L. (2019). Experimental study on the property deterioration of rubber material used as natural rubber bearing under seawater wet-dry cycles. Journal of Vibration and Shock, 38(14), 146-152 + 222 (in Chinese). DOI 10.13465/j.cnki.jvs.2019.14.021.

10. Ma, Y. H., Zhao, G. F., Luo, J. R., Cui, J., Zhou, F. L. (2016). Experimental research on property deterioration of rubber material used as natural rubber isolator for offshore bridges under aging and marine corrosion. Journal of Vibration and Shock, 35(14), 114-122 (in Chinese). DOI 10.13465/j.cnki.jvs.2016.16.019.

11. Liu, J., Li, X. B., Xu, L. K., Zhang, P. (2016). Investigation of aging behavior and mechanism of nitrile-butadiene rubber (NBR) in the accelerated thermal aging environment. Polymer Testing, 54, 59-66. DOI 10.1016/j. polymertesting.2016.06.010.

12. Zhao, J. H., Yang, R., Iervolino, R., Vorst, B. (2015). The effect of thermo-oxidation on the continuous stress relaxation behavior of nitrile rubber. Polymer Degradation and Stability, 115, 32-37. DOI 10.1016/j. polymdegradstab.2015.02.013.

13. Chaabane, M., Ding, N., Zaïri, F. (2021). An approach to assess the thermal aging effects on the coupling between inelasticity and network alteration in filled rubbers. International Journal of Non-Linear Mechanics, 136, 1-13. DOI 10.1016/j.ijnonlinmec.2021.103783.

14. Ulu, K. N., Huneau, B., Gac, P. Y. L., Verron, E. (2016). Fatigue resistance of natural rubber in seawater with comparison to air. International Journal of Fatigue, 88, 247-256. DOI 10.1016/j.ijfatigue.2016.03.033.

15. Huang, J. L., Xie, G. J., Liu, Z. W. (2008). Finite element analysis of super-elastic rubber materials based on the mooney-rivlin and yeoh model. Rubber/Plastics Technology and Equipment, 34, 22-26 (in Chinese). DOI 10.13520/j.cnki.rpte.2008.12.004.

16. Zuo, L., Xiao, F. X. (2008). Analysis of materials coefficient of rubber mooney-rivlin model impacting on the axial stiffness. China Elastomerics, 18, 54-56 (in Chinese). DOI 10.16665/j.cnki.issn1005-3174.2008.03.012.

17. Zuo, L., Xiao, F. X. (2008). One method of determination for material constants of rubber mooney-rivlin model. Machine Building, 527, 38-40 (in Chinese). DOI 1000-4998(2008)07-0038-03.

18. Li, Y. M., Ma, Y. H., Luo, J. R., Zhao, G. F. (2016). The effect of aging on the material constant of the rubber isolator's constitutive model mooney-rivlin. Journal of Vibration and Shock, 35, 164-169 (in Chinese). DOI 10.13465/j.cnki.jvs.2016.16.026.

19. Ma, Y. H., Li, Y. M., Zhao, G. F., Zhou, F. L. (2019). Experimental research on the time-varying law of performance for natural rubber laminated subjected to seawater dry-wet cycles. Engineering Structure, 195, 159-171. DOI 10.1016/j.engstruct.2019.05.101.

20. Yang, X. H., Zhou, C. S., Chang, W. J., Wu, J. (2014). Research on mechanical properties and constitutive model of HTPB rubber inhibitor. Journal of Ballistics, 26, 94-97. DOI 1004-499X(2014)04-0094-04.

21. Sang, J. B., Liu, Y. Y., Xing, S. F., Wang, J. Y., Fu, S. J. (2017). Analysis of hyperelastic mechanical property on rubber like materials under basic load. Journal of Hebei University of Technology, 46, 36-41 (in Chinese). DOI 10.14081/j.cnki.hgdxb.2017.02.007. 
22. ISO 22762-2:2005 (2005). Elastomeric seismic-protection isolators-Part 2: Applications for bridgesSpecifications.

23. ISO 7619-1:2004 (2004). Rubber, vulcanized or thermoplastic —-Determination of indentation hardness—Part 1: Duromerer method (Shore hardness).

24. ISO 37:2005 (2005). Rubber, vulcanized or thermoplastic - Determination of tensile stress-strain properties.

25. Luo, J. (2014). Research on the rubber isolation bearings performance deterioration law under marine corrosion environment (Master Thesis). Guangzhou University, China.

26. ISO 22762-1:2005 (2005). Elastomeric seismic-protection isolations-Part 1: Test methods.

27. JT/T 822-2011 (2011). Lead rubber bearing isolator for highway bridge. The Ministry of Transport of the People's Republic of China. 